\title{
LA TELEBASURA NUESTRA DE CADA DÍA III
}

\section{THE JUNK TV OUR EVERYDAY III}

David Caldevilla Domínguez: Profesor del Área de Comunicación en la Universidad Complutense de Madrid, la Universidad Europea de Madrid y Escuela Superior de Estudios de Relaciones Públicas.

david.caldevilla@ccinf.ucm.es

\section{CURRÍCULUM VITAE}

Diplomado en Magisterio por la Universidad de Zaragoza (España), licenciado en Comunicación Audiovisual por la Universidad Complutense de Madrid (España). Profesor en la Facultad de Ciencias de la Información de la Universidad Complutense. Secretario General del Fórum Internacional de Comunicación y Relaciones Públicas (España) y autor de varios artículos en revistas universitarias. Director de Operaciones de Imagométrica, s.l. y ex directivo de varias firmas españolas del ámbito de la mercadotecnia.

\section{RESUMEN}

En los medios de comunicación el poder se centraliza en unas pocas manos. Lo más importante, es ganar dinero, y ese es el fin de la telebasura. En este artículo repasaremos las cadenas que emiten más telebasura en una evolución diacrónica de la última década del siglo XX y los primeros años del XXI. 


\title{
PALABRAS CLAVE
}

Telebasura - Medios de comunicación - Dinero - Revista

\begin{abstract}
In the media power is centralized in a few hands. The most important thing is to make money, and that's the end of the trash TV. In this article we will review the more trash TV channels that broadcast in a diachronic evolution of the last decade of the twentieth century and the early years of the century.
\end{abstract}

\section{KEY WORDS}

Junk tv - Media - Money - Magazine

\section{ÍNDICE}

1. Introducción

2. Comparativa de parrillas

2.1. Estudio del año 1990

2.2. Estudio del año 1995

2.3. Estudio del año 2000

2.4. Estudio del año 2003

3. Revistas

4. Conclusiones

TEXTO: 


\section{Introducción}

En esta tercera y última, por el momento, entrega, repasaremos las cadenas que emiten más telebasura en una evolución diacrónica de la última década del siglo XX y los primeros años del XXI. Por su especial interés en mantenernos dentro de los estrictos límites del cotilleo intrascendente, de la carnaza barata y del total embrutecimiento de la audiencia, merecen ser incluidas, unas más que otras, sin duda, en nuestra especial Galería de la Fama de la Telebasura Española.

Además, algunas han fomentado la creación de revistas paralelas a las emisiones. Sic transit gloria mundi, ¡ $\mathrm{Si}$ Kempis levantara la cabeza!

\section{Comparativa de parrillas}

En esta fase del informe hemos llevado a cabo una investigación retrospectiva de las parrillas en el 1990, 1995, 2000 y el actual 2003. Los datos se corresponden a la primera semana de noviembre de los años dichos. A través de este análisis queremos confrontar los resultados y hacer una línea de evolución de la telebasura en España.

\subsection{Estudio del año 1990}

Gráfico con las horas emitidas en televisión de telebasura en el año 1990. 

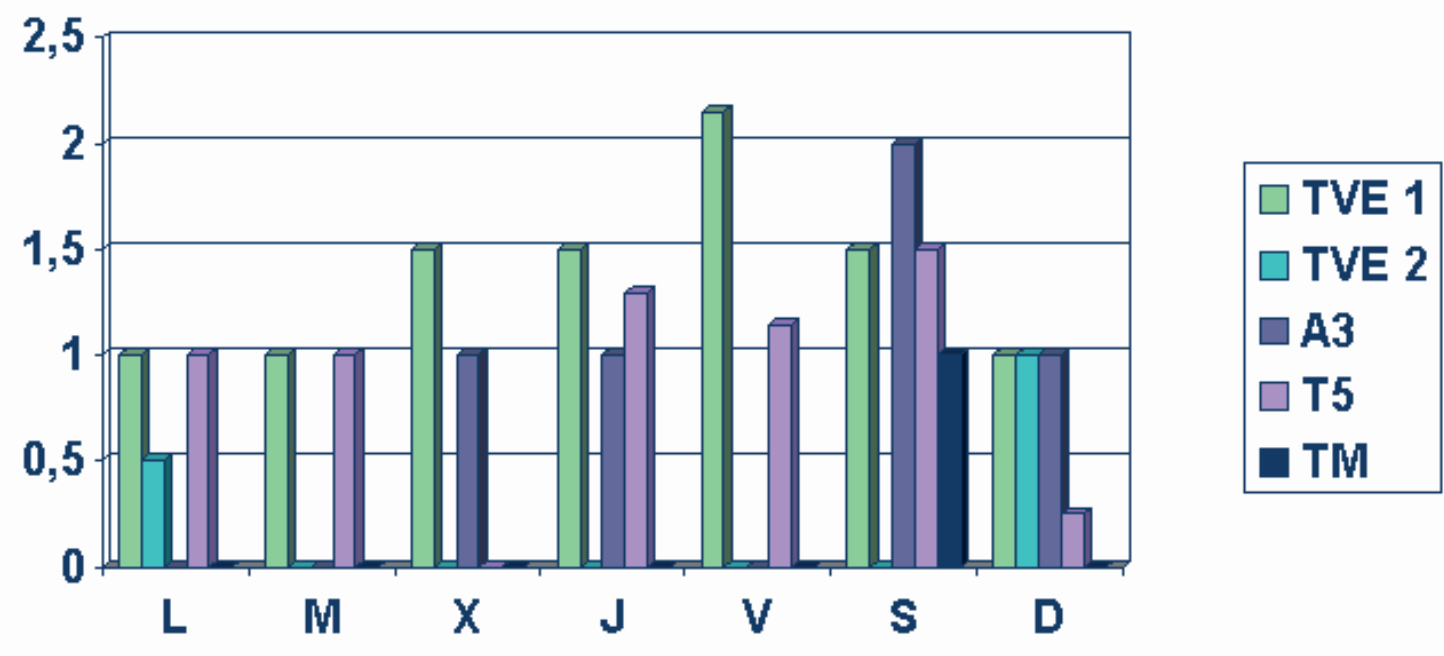

En los años 90 nos ha resultado bastante difícil distinguir qué programas eran de televisión basura y cuáles no, ya que en la actualidad no es fácil encontrar información sobre los mismos. En esta época, había un porcentaje muy pequeño de televisión basura, y la existente aún no está claramente definida, por lo que es difícil realizar un estudio preciso.

Abundan los programas infantiles como es el caso de "Cajón desastre", programa emitido por TVE1 y presentado por Miriam Díaz Aroca.

También son frecuentes programas de humor como "No te rías que es peor", emitido también por TVE1 y "Tutti Frutti", de Raúl Sender y Cruz y Raya.

Nos encontramos en la programación concursos como "Su Media Naranja", presentado por Jesús Puente y emitido por Telecinco.

Abundan un gran número de series como es el caso de "Falcon Crest" y "Corrupción en Miami". 
También encontramos programación más temática como los documentales de La 2, debates de temas concretos con gente especialista (muy alejados de los debates que se dan en los programas actualmente), como es el caso de "De tú a tú", de Nieves Herrero, "Magazines Territoriales", "Entre hoy y mañana", de Jesús Mariñas, programas de deportes (sobre todo en La 2), y programas musicales como "Entre amigos", de José Luís Moreno (programa en el que se mezclaba la música con el humor).

Los programas que hemos considerado "televisión basura" en los años 90, son los siguientes:

"Un día es un día", de Ángel Casas, emitido por TVE1.

"En el calor de la noche", emitido por Antena 3.

"¡Hay, qué calor!", emitido por Telecinco.

"Ésta es su casa", de Mª Teresa Campos, emitido por TVE1.

"Pero... ¿Esto qué es?", de Carlos Mata, Jacqueline de la Vega y Elisa Matilla, emitido en TVE1

"Hablando se entiende la gente", de Telecinco.

"Te veo de noche", de TVE1

"Telecinco, ¿Dígame?", de Laura Valenzuela.

"El salero", de Canal Plus. 
"Sara y punto", de Sara Montiel, emitido en TVE2.

"Ricos y Famosos", en Antena 3.

"Chispa de famosos", en Telecinco.

CONCLUSIÓN: podemos concluir con que, en 1990, sólo había programas de televisión basura de manera esporádica y en un porcentaje muy inferior al actual.

DATOS PORCENTUALES: para realizar el estudio, hemos tomado como muestra para cada año la primera semana del mes de noviembre, para calcular el número de horas que cada cadena dedicaba a la emisión de programas de televisión basura.

El número total de horas de telebasura en la semana del 1 al 7 de noviembre de 1990 es de 23 horas y 40 segundos, según nuestro criterio.

\subsection{Estudio del año 1995}

Gráfico con las horas emitidas en televisión de telebasura en el año 1995. 


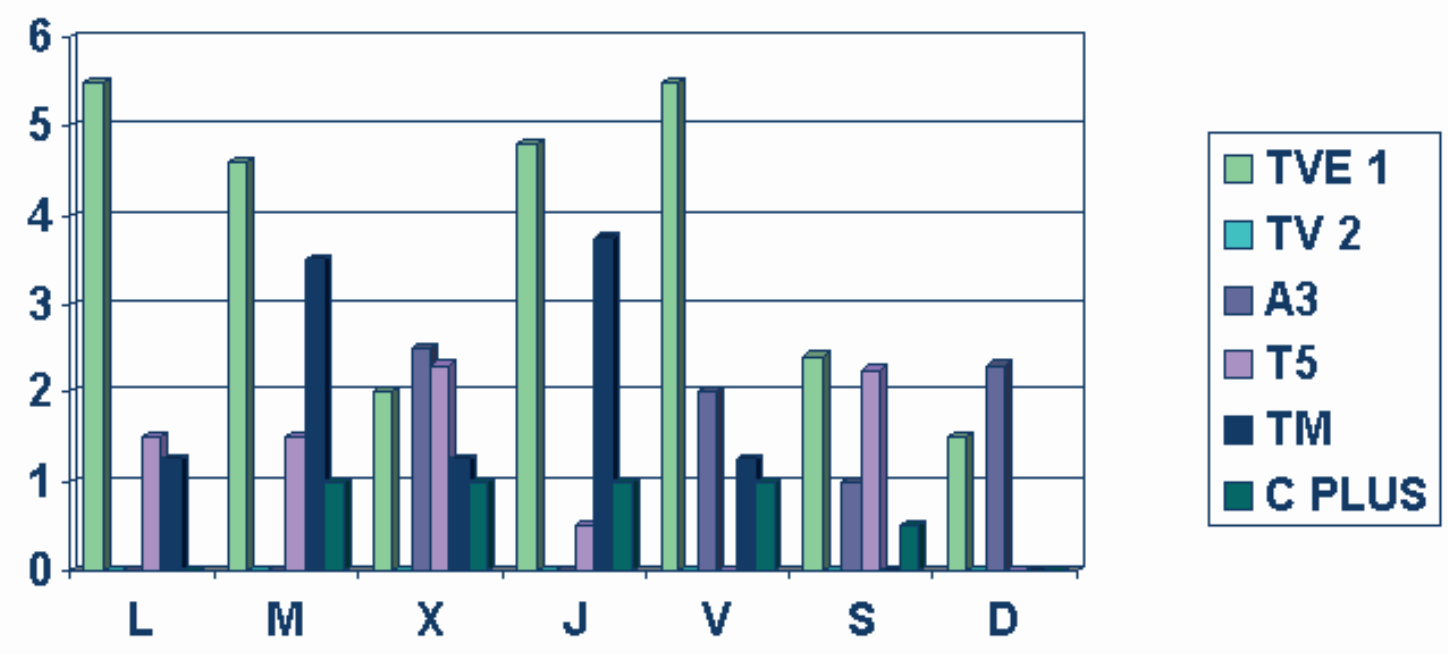

Nuestros estudios realizados sobre la programación de la televisión del año 1995 demuestran un incremento considerable de la programación basura con respecto a los comienzos de los años $90^{\prime}$.

La televisión en este año se centra más en la emisión de otro tipo de programas como las series, donde destacan " Falcon Crest" o "Alf " entre otras, sin contar claro con las telenovelas que ocupan desde hace muchos años una franja horaria determinada e inamovible y con una audiencia sostenida y equilibrada a lo largo de los años. Entre las novelas más destacadas están " Cristal" o " Topacio".

Otro tipo de programas que tenían gran audiencia y que se mantuvieron en la programación son los concursos como por ejemplo "Su media naranja", presentado por Jesús Puente y estrenado en Antena 3 en los años 90’.

Por último vamos hacer un breve repaso de la programación consolidada como telebasura en sí, donde destacan programas como: 
- "El programa de Ana" ---- Programa de debate donde acuden participantes para contar sus vivencias sobre un tema concreto. Para ello la presentadora puede encauzar la declaración del participante haciéndoles preguntas concretas.

- "Esta noche cruzamos el Mississippi"---- Programa consolidado como un Talkshow emitido de lunes a viernes a partir de las 00.00. Este programa puede considerarse como el predecesor de " Crónicas marcianas" emitido poco después por el mismo canal Tele5.

- "QMQ" (Qué me dices) ---- Programa de sobremesa presentado por Belinda Washington y emitido de lunes a viernes de 15.00 a 15.30. En este programa se tratan las principales noticias del día relacionadas con el mundo del corazón y tratadas con cierto punto de humor. Fue un programa líder de audiencias por lo que posteriormente se decidió realizar una revista siguiendo la misma línea informativa, que salía a la venta los jueves. Revista y programa convivieron durante mucho tiempo pero, posteriormente, el programa de dejó de emitir aunque la revista sigue dando todavía mucho que hablar.

- Por último, cabe tratar los magazines de actualidad encargados de informar al espectador de las noticias de la actualidad pero desde una perspectiva más periodística. Un ejemplo de ellos son " Pasa la vida " presentado por María Teresa Campos y emitido de forma matinal de lunes a viernes en TVE1. En esta línea, años más tarde, se realizará el programa "Día a día " también presentado por María Teresa Campos.

- Otro tipo de Magacine es "A toda página", que es considerado un programa de actualidad donde tratan noticias sucedidas a lo largo de toda la semana. 
Como balance general del año 1995 podemos observar como, poco a poco, va aumentando la emisión de programas considerados como basura, ya que los productores ven como son los programas más vistos y comentados a pesar de todas las críticas que reciben.

Esto será el origen de lo que se emitirá en el año 2000, cuando se multiplican los programas del corazón expuestos desde diferentes perspectivas.

\subsection{Estudio del año 2000}

Gráfico con las horas emitidas en televisión de telebasura en el año 2000.

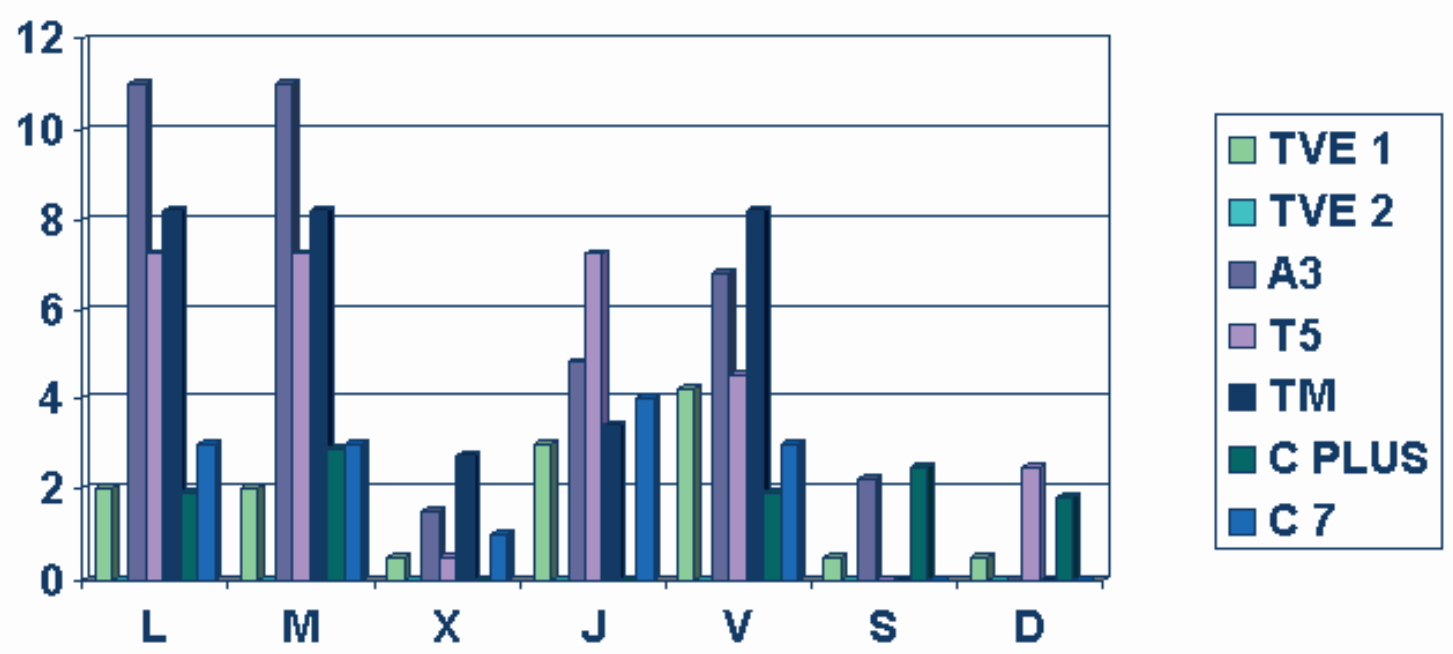

En el año 2000 se ve un incremento notable de los programas de televisión basura.

Destacan programas como: 
"Corazón de Otoño", "El Informal", "Sabor a Ti", presentado por Ana Rosa Quintana, "Día a día", de Ma Teresa Campos, "Mamma Mía", de Víctor Sandoval y Patricia Pérez," Con T de Tarde", de Terelu Campos.

Además, en El año 2000 despega el Canal 7 con programas de televisión basura como "Corazón del Milenio".

En el 2000, ya existen nuevos estilos de programas " basura" entre los que destacan:

"Crónicas Marcianas", de Javier Sardá y Boris Izaguirre.

Programas que siguen el mito del panóptico, una cárcel en la que el preso siempre se siente vigilado, pero en la cual el prisionero no puede ver a sus carceleros:

El precursor de estos programas fue "Gran Hermano", emitido por primera vez en el 2000, a partir del cual se lanzaron a la emisión otras variaciones panópticas como:

"Operación Triunfo": concurso que comenzó siendo un programa artístico y que se ha ido convirtiendo en un programa más de televisión basura.

"Supervivientes": emitido en el 2000-2001 por Telecinco. Concurso donde los participantes se enfrentaban a carencias de alimentos y de comodidades de la civilización.

"El Bus": programa emitido en el 2000 por Antena 3, pretendiendo competir con la segunda edición de Gran Hermano. 
"Confianza Ciega": programa emitido por Antena 3. La novedad que introdujo respecto a Gran Hermano fue que los concursantes eran parejas y que se enfrentaban a pruebas de fidelidad.

También hay que destacar en el 2000 los programas que se crearon en Canal Plus para cubrir los huecos de la programación y para evitar quejas al emitir todo en cerrado. Entre ellos podemos destacar: "Zap... zap... zapin", "Pin... pan... Plus", "Lo más Plus".

CONCLUSIÓN: podemos concluir con que, en el año 2000 hay un incremento considerable de los programas de televisión basura. Además, la televisión basura se establece como un nuevo género, apareciendo de manera muy continuada en casi todas las cadenas. En el 2000 se produce el boom de la telebasura con la aparición de Gran Hermano.

DATOS PORCENTUALES: el número total de horas de telebasura del 1 al 7 de noviembre del año 2000 es de 133 horas y 30 minutos, según nuestro criterio.

\subsection{Estudio del año 2003}

Gráfico con las horas emitidas en televisión de telebasura en el año 2003. 


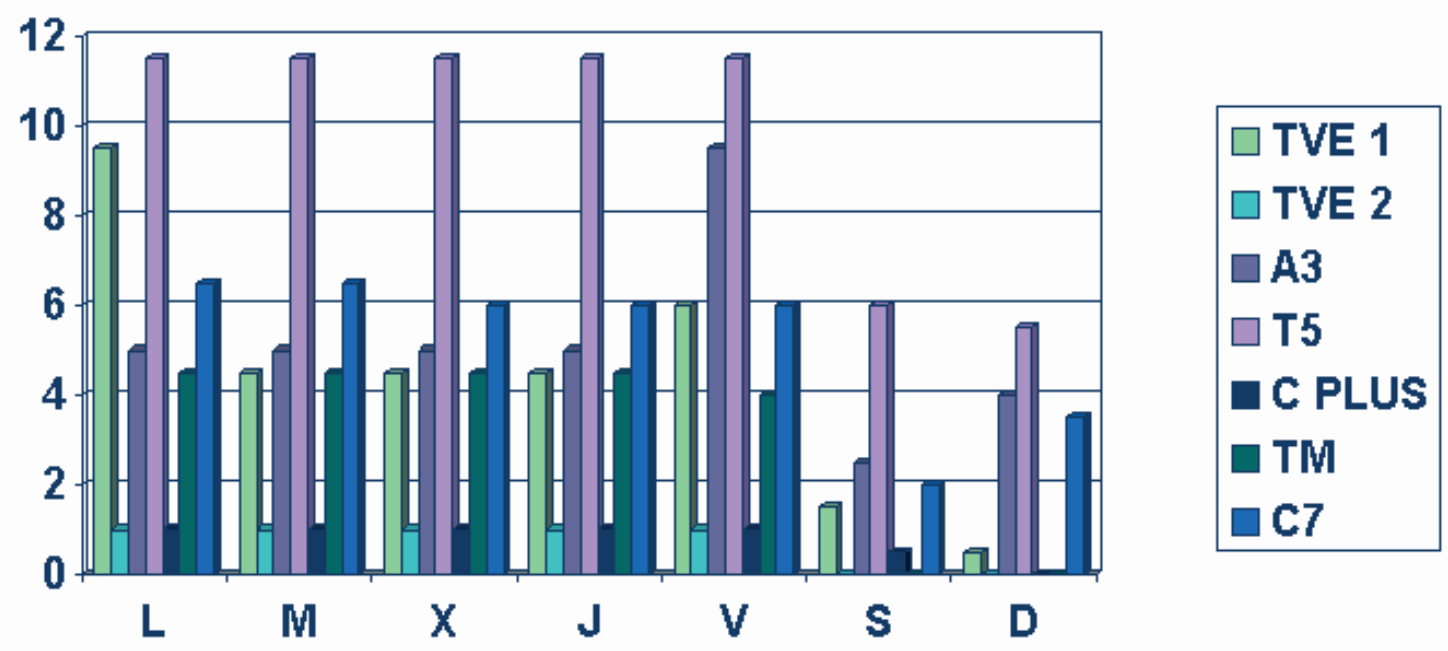

Durante el año 2003, y como era de esperar, ha habido un gran aumento de la programación basura en todas las principales cadenas de televisión como Antena 3, Tele5 o incluso Telemadrid, exceptuando La 2 y Canal + que continúan en su línea de crear una televisión mas educativa y entretenida sin explotar el morbo que dan las noticias del corazón.

El hecho de que Canal + y La 2 no emitan tanta programación basura como el resto de las cadenas ha hecho que disminuya su porcentaje de audiencias diarias, ya que los magazines, los debates y talkshow, donde son llevados gran cantidad de invitados para que discutan con otros, sacando sus trapos sucios sólo por dinero, está a la orden del día.

De esta manera la principal cadena de televisión y líder de audiencias, con una media de porcentaje diario de 24.1 es TVE1, cadena con una amplia programación. Sus programas telebasuras más vistos a lo largo de todas las semanas son "Gente" que es un programa dividido en dos bloques, uno dedicado a noticias ocurridas en España de interés general como muertes, asesinatos, robos o distintos sucesos y el segundo bloque dedicado al mundo de los famosos dónde sacan a la luz nuevos compromisos, 
separaciones, nacimientos....La emisión de este programa es de lunes a viernes a partir de las 20 horas.

Otro programa que ha tenido una gran expectación para TVE1 ha sido "Operación Triunfo", que es un concurso donde los participantes son educados y amaestrados en el arte del espectáculo y la canción, para ello los concursantes debían permanecer una serie de meses en la Academia, con profesores expertos en la materia para, cada semana, deleitar al público con sus canciones, que serán los que elijan quien debe abandonar la Academia. El programa va ya por su tercera edición y, a decir verdad, va perdiendo cada vez más expectación, por lo que los productores del programa han decido acortar su duración. A pesar de todo Operación Triunfo es uno de los programas más vistos por la audiencia ya que no es emitido sólo una vez a la semana, sino que, mediante una serie de resúmenes diarios emitidos tanto en TVE1 como en La 2, van conociendo la actualidad y las novedades ocurridas en la Academia.

El último programa de telebasura emitido por TVE1 que vamos a tratar es "Corazón de Otoño" (En este caso es de otoño porque hemos estudiado la primera semana de Noviembre pero el nombre del programa va variando según la estación). Este programa está formado por una serie de bloques que van dando las noticias más destacadas del día, ya que es emitido de lunes a viernes de 14.30 a 15.00 y presentado de Anne Igartiburu. Y si esto es así de Lunes a Viernes, los fines de semana Anne pasa el relevo a Corazón Corazón que sigue la misma línea que el anterior. Este programa tiene menos audiencia que el anterior ya que los fines de semana, al haber más variedad de programación, la audiencia se divide.

El resto de los programas emitidos por TVE1 de mayor audiencia suelen ser series tales como "Smallville" o "Cuéntame cómo pasó" (Serie de gran audiencia por su fondo histórico, ya que reconstruye la España de la época de posguerra de una 
manera muy gráfica y divertida, con la mayor verosimilitud posible). "Gata Salvaje" (Novela de sobremesa que cuenta los líos que suceden en una casa, todos relacionados con asuntos amorosos, desengaños, celos....).

Los fines de semana la televisión se hace cinéfila, es decir, lo que más se emiten son películas de diferente índole.

La segunda cadena líder de audiencias, con una porcentaje diario de 23.1, es Tele5. Ésta es una de las cadenas de televisión considerada como la que emite mayor tiempo de programación basura. Sus programas más destacados son:

"Pecado Original" que es un programa actualidad del corazón donde, de forma divertida, nos presentan las diferentes noticias. La mezcla de humor y noticia hacen de pecado original una química explosiva. A favor de este programa hay que decir que su inmejorable franja horaria permite a este programa ser uno de los más vistos.

Otro programa que cabe destacar es "Aquí hay tomate", programa emitido de Lunes a Viernes de 15.30 a 16.30 y presentado por Jorge Javier Vázquez y Carmen Alacaide, donde presentan los noticias rosas más destacadas y los mismos presentadores pueden comentarlas. Así mismo se abre un teléfono para que los aludidos pueden llamar y entrar en antena para defenderse de lo dicho. Es un programa de mucha polémica pero muy visto por la audiencia.

"Gran Hermano", otro programa de gran audiencia a pesar del número de ediciones (5), que sigue manteniendo al público pegado a su asiento.

Los jueves, cita con Mercedes Milá y con la colaboración de Jorge Fernández además con todos los concursantes expulsados de la casa para dar a conocer la nueva lista de nominados o comunicar a la casa la nueva expulsión. 
"El debate" emitido los domingos a partir de las 22.00 y presentado por Jesús Vázquez es otro programa dedicado a Gran Hermano, en él ex concursantes de la casa dan su opinión abiertamente sobre los sucesos, comportamientos y hechos en la casa.

Por último, y no por ello menos importante, está "Crónicas Marcianas". Este programa, emitido en la madrugada y presentado por Javier Sardá y Boris Izagirre muestra una mirada más crítica sobre Gran hermano, para ello cuenta con concursantes expertos de otras ediciones que dan su opinión sobre los hechos.

Otro programa de gran audiencia es "Salsa Rosa" que puede ser considerado como la explosión del morbo televisivo, donde gran cantidad de invitados van al programa a contar sus intimidades, para que los colaboradores fijos que hay en el programa, como Coto Matamoros, Carmen Hornillos o Lidia Lozano, saquen punta a todas sus frases y se pueda discutir con el fin de atraer al espectador. Todo ello por una suma elevada de dinero.

Pero Además de estos programas Tele5 es una de las cadenas más vistas por series de gran expectación como "Los Serrano" o "C.S.I" (Serie de investigación criminal).

Los fines de semana, como todas las cadenas de televisión, Tele5 se dedica más al cine manteniendo una pequeña parte para el público infantil.

En tercer lugar cabe hablar de Antena3 con un porcentaje de audiencia diario de un 20.2 .

El programa más visto, sin lugar a dudas, en esta cadena es " Los Simpson", que es una serie de dibujos animados emitida todos los días de 14.00 a 15.00 para todos los públicos. Tiene unas grandes dotes de humor y permite pasar un rato divertido con 
las aventuras y peripecias de una familia que no llega a congeniar en el mundo en el que vive.

El cine en Antena3, al igual que las series, ocupa una parte muy importante de la parrilla de la programación que se llevan los mayores share de audiencias.

El resto de las cadenas tanto las autonómicas, como La 2 y Canal+ tienen porcentajes de audiencias muy inferiores a las demás y sus programas no llegan ni siquiera a ocupar un puesto entre los 10 programas más vistos, por lo que esta información nos sirve para ver como las cadenas que más telebasura emiten, son las que mayor porcentaje de audiencias tienen, ya que ahora mismo el morbo es lo que mueve a la televisión, y no nos podemos quejar por ello, por que somos nosotros mismos los que lo estamos consiguiendo con nuestra visión de los mismos.

\section{Revistas}

\section{REVISTAS PERIÓDICAS SURGIDAS A PARTIR DE UN PROGRAMA DE}

\section{TELEBASURA}

Son bastantes los programas del corazón que han dado origen a una publicación periódica: Gran Hermano, Operación Triunfo, Qué me dices y Sabor a Ti (el programa de Ana Rosa Quintana).

Sabemos que los programas de gran audiencia, y concretamente el popular "Gran Hermano", llenan los distintos espacios televisivos dedicados al corazón de contenido. Pero no sólo eso, sino que también plagan las revistas rosas que se venden en España. 
Nosotros vamos a hablar de una frontera más que han sido capaces de superar: la de dar origen a una publicación por sí mismos, a revistas que se dedican única y exclusivamente a ellos.

GRAN HERMANO: Revista Oficial.

- Revista que cuesta 1 euro, de publicación quincenal.

- Dedicada exclusivamente a Gran Hermano.

- Está dividida en varias secciones-

- Gente GH- en la que se hace un resumen de cosas curiosas que han sucedido dentro de la casa y declaraciones de personas relacionadas con el programa (antiguos concursantes, familiares de concursantes, etc.)

- Sección de entrevistas- Normalmente a familiares de los concursantes que están ahora en la casa.

- Espacio dedicado a analizar los nominados y quiénes les nombraron.

- Artículos escritos por antiguos participantes.

- Colaboraciones de personajes como Mercedes Milá, presentadora del programa de televisión, que opina sobre la situación en la casa.

- Reportaje turístico relacionado con una ciudad concreta- País natal de algún personaje del programa. 
- Sección de Moda- Publicidad de marcas de ropa- relacionada con algún personaje de la casa.

- Una sección donde se detallan día a día los sucesos que ocurren en la casa.- Día a Día.

- Sección de Cartas a la redacción para que opinen los lectores y un test sobre el programa.

- Sección de pasatiempos.- Sopa de letras, crucigrama, diferencias.

- Horóscopo.

- Programación semanal.

- Reportajes sobre ex concursantes.

- Resumen sobre otras noticias del corazón no relacionadas con el programa.

- Sección de citas, curiosidades y variedades- Quién dijo qué.

- La revista de Gran Hermano está financiada con publicidad que corre a cargo del grupo Hachette Flipachi e Interdecó.

- Como redactores colaboran personas como Carmen Hornillos (actualmente trabaja también en Canal 7, junto a Carlos Ferrando en "Corazón del Milenio") o Cristina Fernández.

- La directora de la revista es Pilar García Vázquez. 
- La revista la edita Multiediciones Universales S.L.

- Se edita bajo licencia de Gestevisión Telecinco/Zeppelín.

\section{Conclusiones}

Tras terminar nuestra investigación, hemos llegado, en primer lugar, a la conclusión clara y firme de que en los medios de comunicación el poder se centraliza en unas pocas manos. Es un mercado muy duro, controlado por grandes magnates, donde lo más importante, es ganar dinero. Creemos que la telebasura sólo tiene un fin, que es el monetario, y que sobrevive gracias a la protección que les ofrece su grupo empresarial, ayudándolo desde otras fuentes de información que están en su poder. Es importante saber quién es el jefe de tal o cual medio a la hora de relacionar ciertas informaciones.

Quizá se deriven de este hecho las dificultades que hemos encontrado a la hora de conseguir la documentación al solicitarla en las cadenas de televisión. Al contactar con los diferentes programas, hemos percibido cierto secretismo en algunos aspectos de la profesión y de los espacios que elaboran. Con esto, parecen querer dar una imagen positiva e idealizada sobre su forma de trabajar, que no sabemos si se corresponde o no con la realidad, ya que no hemos recibido la información solicitada.

Creemos necesario comentar también los problemas que nos hemos encontrado a la hora de seleccionar la información, puesto que hay gran parte de documentación, pero no toda ella es fiable, y fue necesario identificarla y renunciar a ella.

Por otra parte, y ligada a la idea de la documentación, no tenemos más remedio que denunciar el hecho de que apenas existe bibliografía específica y válida sobre el tema. Realmente, la única que nos ha servido y que por tanto consideramos correcta y útil 
es la que conseguimos en el transcurso de nuestra visita a la cadena privada Telecinco: "Anuario 2003".

Durante nuestra investigación, se han ido conformando en nosotros una serie de ideas que dan explicación a la producción y emisión tan abundante de programas de telebasura. Nuestra teoría es que las cadenas se lanzan a realizar estos programas porque, por una parte, son productos muy baratos. Por otra, sus formatos no son muy variados y son fácilmente imitables entre sí, es decir, no exigen de los responsables mucho esfuerzo en cuanto a imaginación y profesionalidad. De hecho, casi todos los programas que encontramos hoy en día en la televisión son copias de programas que llevan funcionando bastante tiempo anteriormente y que tienen éxito. Y, fundamentalmente, hay que comentar que otra de las razones es que estos espacios mantienen unos niveles de audiencia que, no son los mejores, pero si son bastante aceptables. Por otra parte, también encontramos otros programas que nacieron con unas características alejadas de la telebasura pero que se han ido transformando con las demandas del mercado y por tanto han ido llegando a una situación de decadencia visible con claridad actualmente, como es el caso de "CRÓNICAS MARCIANAS", de Telecinco. Para reflejar esto, remitimos a un artículo aparecido en el periódico 20 Minutos:

\section{"CRÓNICAS MARCIANAS"}

ANTES: 1500 actores, modelos, cineastas... han participado como invitados en Crónicas Marcianas desde su estreno el 8 de septiembre de 1997. Entre ellos Cindy Crawford, David Copperfield, Enrique Iglesias, Ricky Martín... 
Hace años, el inefable Galindo era quien ponía algo de cordura en el disparatado Marte. Junto con él, revolviéndolo todo, estuvieron colaboradores cómo Fuentes, Mariano Mariano, Paz Padilla, Rosario Pardo...

Como contertulios estuvieron, entre otros, Ivonne Reyes, Ramoncín, Loles León, Javier Nart, la sexóloga Carmen Vijande...Todos ofrecían su particular punto de vista sobre la actualidad.

AHORA: Últimamente se entrevista a personajes como Nuria Bermúdez, Mila Ximénez, María José Galera, Yola Berrocal, Dinio, Támara, Pocholo Martínez Bordiú, Noemí, Sonia Monroy, Isabel Madow...

Boris Izaguirre es quien hoy en día introduce en la mesa los comentarios irónicos y originales. Junto con él están el impagable actor Carlos Latre, Rocío Madrid, Javiera Cárdenas y Xavier Deltell.

Coto Matamoros, los grandes hermanos Marta, Kiko, Raquel, Jorge Berrocal, Aída..., Silvia Fominaya, Mari Cielo Pajares, Antonio David... animan ahora el espectáculo con sus ocurriencias.

A la hora de reflexionar sobre esto, llegamos a la controversia que siempre se plantea cualquier investigación de este tipo y que se concreta con la pregunta: ¿Es el público el que demanda estos programas o son las televisiones las que obligan a la gente a aceptar este tipo de programación ya que no ofrecen nada más allá de ella?

Una posible explicación de este problema, según nuestro punto de vista, está en la evolución de la propia sociedad. Las personas somos sociables por naturaleza y encontramos placer en saber cosas de los demás. En nuestras ciudades actuales, tan extensas y superpobladas, es muy difícil mantener relaciones sociales como ocurría 
antiguamente en los núcleos rurales pequeños. Por ello, la gente se recluye cada vez más en sus casas, sola, y utiliza la telebasura como un medio para tener contacto humano con los otros. Este fenómeno tiene un nombre "VENTANA A LA REALIDAD", y un ejemplo de esto es el conocido espacio "Gran Hermano", también conocido como TELEREALIDAD.

Realmente, no sabemos si esto es la causa o el efecto, ambos lo son. Por una parte, la gente se refugia en la telebasura porque se relaciona menos con las personas de su entorno, pero, por otra parte, al ver tanta telebasura, también se implica menos en su contacto con el resto de las personas de su entorno.

La estructura de estos programas permite que con sólo verlo en un corto espacio de tiempo te enteres porque son reportajes de minuto / minuto y medio, de forma episódica y muy repetitivos, por lo que no necesitan un alto grado de atención ni llevar un seguimiento continuado ya que en cualquier momento te puedes volver a enganchar. Es el efecto narcotizante, vemos la televisión sin pensar en nada ni tener que analizar lo que se está viendo.

Hemos comprobado que en los inicios de los programas telebasura los presentadores y colaboradores eran profesionales del mundo del periodismo y, poco a poco, la gente que habla no tiene profesión, ni estudios reconocidos.

En el último año / año y medio ha aparecido un fenómeno llamado "CASPA", son personajillos sin oficio que saltan a los platós de televisión contando, en la mayoría de las ocasiones, aspectos de la vida privada de personajes famosos que sí lo son. Esto demuestra, una vez más, la escasa calidad de estos programas tanto desde el punto de vista técnico, como profesional y moral. 
Hemos observado tras el estudio de las distintas cadenas que hay un mayor número de horas dedicadas a la telebasura en televisiones privadas en abierto. En la televisión pública hay menos dedicación a esta clase de programas y se trabaja de una manera más profesional, últimas noticias indican que el actual gobierno quiere eliminar los últimos resquicios de telebasura en la cadena autonómica de Madrid RTVMA. Por último, decir que en los canales de pago la telebasura es casi inexistente, sólo se utiliza para rellenar, suelen ser zapping de otras cadenas (zap, zap, zapping y el más te vale), por lo que llegamos a la conclusión de que, en realidad, la gente paga por ver una televisión de calidad, y dentro de esta definición no podemos incluir a la telebasura, si lo fuera estarían incluidos en las parrillas de los canales de pago. 